Mots. Les langages du politique

$93 \mid 2010$

Figures et filiations dans le discours politique latinoaméricain

\title{
Michael Rinn éd. Émotions et discours. L'usage des passions dans la langue
}

Éléonore Yasri-Labrique

\section{(2) OpenEdition}

\section{Journals}

Édition électronique

URL : https://journals.openedition.org/mots/19834

DOI : $10.4000 /$ mots. 19834

ISSN : 1960-6001

Éditeur

ENS Éditions

Édition imprimée

Date de publication : 1 juillet 2010

Pagination : 145-148

ISBN : 978-2-84788-231-5

ISSN : 0243-6450

Référence électronique

Éléonore Yasri-Labrique, « Michael Rinn éd. Émotions et discours. L'usage des passions dans la

langue », Mots. Les langages du politique [En ligne], 93 | 2010, mis en ligne le 01 octobre 2012, consulté le 23 avril 2022. URL : http://journals.openedition.org/mots/19834 ; DOI : https://doi.org/10.4000/ mots. 19834 


\title{
Comptes rendus de lecture
}

\author{
Émotions et discours. L'usage des passions dans la langue \\ Michael Rinn éd. \\ 2008, Rennes, Presses universitaires de Rennes (Interférences), 371 pages
}

L'ouvrage intitulé Émotions et discours, dirigé par Michael Rinn, professeur en sciences du langage à l'université de Bretagne occidentale, est un recueil de vingt-et-une contributions signées de grands noms de l'analyse du discours : Ruth Amossy, Marc Angenot, Patrick Charaudeau, Christian Plantin... Il est divisé en quatre parties équilibrées qui mettent chacune l'accent sur une dimension particulière du pathos, à propos duquel le Dictionnaire d'analyse du discours nous rappelle les points suivants : «Dans l'usage courant, le mot "pathos" est pris actuellement au sens de débordement émotionnel, généralement manquant de sincérité, acception qui n'affecte pas son dérivé "pathétique". En rhétorique, le terme renvoie à l'un des trois types d'arguments, ou preuves, destinés à produire la persuasion. $»^{1} C^{\prime}$ 'est donc bien de la mise en mots et de la mise en scène des émotions à des fins argumentatives qu'il s'agit dans cet ouvrage qui explore plusieurs facettes du registre passionnel à travers des synthèses théoriques ou des études de cas.

Ce recueil de contributions internationales et transdisciplinaires, où l'histoire, la politique et la sociologie rejoignent la linguistique, la littérature et la communication médiatique, mais où dominent constamment les interrogations concernant la rhétorique dans ses ressorts a priori les moins rationnels, s'ouvre par une introduction de Michael Rinn qui l'inscrit d'entrée de jeu dans une dimension principalement éthique. Dès les premières lignes, il précise en effet : «La réflexion méthodologique paraît urgente, compte tenu de la montée en puissance des nouveaux discours identitaires, communautaristes, négationnistes et racistes. Empruntant aux passions communes, ces discours persuasifs cherchent à réduire la pluralité des valeurs culturelles nécessaires à la vie en société. » (p.13) Il détaille ensuite le contenu des quatre parties constitutives de cette réflexion cruciale. "La pensée pathétique» s'interroge sur les modèles divergents qui, de l'Antiquité à nos jours, ont émergé pour traiter la

1. Patrick Charaudeau, Dominique Maingueneau éd., Dictionnaire d'analyse du discours, Paris, Le Seuil, 2002, p. 423. Les auteurs soulignent. 
question des émotions dans le discours. La deuxième partie, "Les arguments du pathos », démonte le double fonctionnement de la logique passionnelle et analyse le défi que constitue le déni argumenté. Dans "La poétique de l'action », centrée sur des recherches stylistiques, le lecteur est invité à s'interroger sur les aspects esthétiques des constructions pathémiques. Enfin, la dernière partie, la plus longue avec six chapitres au lieu de cinq pour les trois premières, s'intitule «Pour une herméneutique du pathos » et s'assigne pour objectif primordial de "proposer les concepts d'une sémiologie du pathos afin de contribuer à la critique renouvelée de la culture sociale » (p. 17).

Il n'importe pas ici de faire un résumé de chaque contribution, si intéressante soit-elle intrinsèquement. Mais il parait essentiel d'insister sur le cheminement rigoureux de la pensée des chercheurs qui non seulement proposent des analyses de discours pointues et étayées, mais également se positionnent en citoyens engagés face aux dangers qu'entrainent les dérives passionnelles de la communication langagière. Ce recueil est également un voyage diachronique qui nous emmène d'abord à Rome, plus de vingt siècles en arrière, pour une plongée dans la rhétorique grecque puis latine, mettant en lumière ce que Fernand Delarue nomme «l'union de la passion et de la lucidité» (p. 35), et finit par nous conduire au cœur de la presse contemporaine, entre New York et Paris, à l'occasion de deux événements qui ont marqué le début du troisième millénaire, les attentats du 11 septembre 2001 et la mort du leader palestinien Yasser Arafat, le 11 novembre 2004. Ces interdiscours médiatiques sont tous deux étudiés dans leurs aspects iconographique et langagier. La première de ces analyses consacre une réflexion capitale à «l'esthétisation de l'événement par l'image [ou] par le témoignage» (p. 341-343); la deuxième s'intéresse davantage aux relations complexes entre signification, énonciation et image (p. 354). Ainsi, Aurélie Lagadec souligne notamment que «par cette volonté d'exacerber la dimension agonique de la force pathétique de l'événement, à travers l'esthétisation sous couvert d'éthique, les médias ouvrent la porte à un espace de controverse» (p. 349), tandis que Louis Panier propose une approche sémiotique qui pose «la question de l'émotion produite et partagée» (p. 359).

C'est aussi cette interrogation qui sous-tend, certes d'une manière différente, le travail de Raphaël Michelli à propos de la construction argumentative des émotions en relation avec le débat sur l'abolition de la peine de mort en 1908. Pour lui, «l'analyste doit jauger les effets d'un appel à l'émotion, et ceci en regard du déroulement idéal d'une argumentation tel que le fixe, de façon normative, un modèle de dialogue» (p.128; Michelli souligne). Déjà complexe par essence, la tâche du chercheur atteint une sorte de difficulté paroxystique, mais en même temps une ampleur primordiale, quand il s'attelle non plus à des sujets que l'on pourrait qualifier de sensibles, mais à des mises en discours de vécus relevant de l'indicible. C'est ce que font au centre de l'ouvrage Philippe Mesnard, d'une part, et Michael Rinn, d'autre part. Le premier explore, dans 
"L'ambivalence du vide, entre Giorgio Agamben et Benjamin Wilkomirski », la «réception pathétique » et les effets triangulaires sur les auteurs, leurs textes et les lecteurs, d'une écriture "en phase avec l'époque hautement mémorielle qui est la nôtre, où le régime esthétique dominant est celui d'un pathos exclusif et désarticulé des traditions de la rationalité » (p.187); le second aborde, dans "Critique des réfutations négationnistes », une thématique et des questionnements semblables qui le conduisent, après une étude des stratégies argumentatives en circulation sur le Net, à se demander : «Que restera-t-il de l'Autre et du moi dans ce vide de sens?» (p. 202). De la sorte, ce recueil de contributions apporte des éléments de réponse concernant l'usage des passions dans la langue, que ce soit à propos d'œuvres littéraires ou philosophiques, ou encore de discours politiques, médiatiques ou sociaux à la disposition du grand public, mais surtout invite le lecteur à se défaire d'idées reçues et à s'interroger sur les enjeux profonds de l'implication, l'implémentation parfois, des émotions langagières qui affectent à la fois émetteurs et destinataires.

Comme dans son ouvrage Les médias et l'information. L'impossible transparence du discours ${ }^{2}$, Patrick Charaudeau se penche à nouveau sur l'asymétrie qui existe entre le pôle de production et le pôle de réception d'un message. À la recherche des « traces sémiologiques des émotions» (p. 50), il note que certains termes sont aptes à susciter des sentiments de peur, d'indignation ou de colère, mais que « tout dépend de l'environnement de ces mots, du contexte, de la situation dans lesquels ils s'inscrivent, de qui les emploie et qui les reçoit » (p. 51). Dans cette contribution, c'est au discours politique qu'il s'intéresse, en particulier à «la mise en scène du pathos dans le discours populiste» (p. 54). Il identifie certains aspects de la dramaturgie discursive caractéristique des propos de leaders nationalistes (la dénonciation d'une situation de déclin, la désignation d'une source du mal, l'auto-attribution du rôle de sauveur, l'exaltation des valeurs liées aux origines...), mais conclut que « le recours aux effets pathémiques est constitutif du discours politique» (p. 57). Dans une certaine mesure, Marc Angenot lui fait écho lorsqu'il étudie le ressentiment à travers plusieurs types de discours politiques, de droite comme de gauche, et s'interroge sur «l'essence du populisme » : il propose alors « le paramètre du ressentiment comme critère propre de la notion de populisme » (p. 94) et distingue les amertumes populaires spontanées des rancœurs sélectionnées, orientées et entretenues par les doctrinaires de tels mouvements. Il prévient : «On peut prévoir le triomphe de l'idée même de la droite nationale [...], idée qui s'est revêtue de nos jours des haillons du progressisme : quelle est cette idée ? Que la valeur éthique fondamentale est la culture et les traditions d'un groupe déterminé et que celles-ci engendrent des droits collectifs suprêmes. » (p. 96-97) À

2. Patrick Charaudeau, Les médias et l'information. L'impossible transparence du discours, Louvain-la-Neuve, De Boeck, 2005. 
l'heure où, d'un côté, les polémiques alimentent le débat sur l'identité nationale lancé par le gouvernement français et où, de l'autre, les dernières élections régionales confirment la présence de partis populistes locaux ou nationaux, cet avertissement est davantage qu'une remarque d'ordre sociolinguistique, c'est un véritable signal d'alarme.

Émotions et discours est donc bien un ouvrage phare dans la réflexion sur l'usage des passions au sein de multiples univers de discours. Bien qu'on ne puisse en aucun cas le considérer comme anodin, qu'on s'intéresse à des périodes de l'Histoire révolues ou à des œuvres littéraires ayant plus ou moins marqué les consciences collectives, le jeu sur les émotions à travers le langage n'est sans doute jamais aussi périlleux que dans les discours politiques explorés ici par plusieurs contributeurs.

Éléonore Yasri-Labrique

Université Montpellier 3, ARSER-DIPRALANG

yeleonore_2000@yahoo.fr

La notion de «formule» en analyse du discours.

\section{Cadre théorique et méthodologique}

Alice Krieg-Planque

2009, Paris, Presses universitaires de Franche-Comté, 144 pages

Le dernier ouvrage d'Alice Krieg-Planque est de ceux qui fournissent à l'analyse du discours actuelle un appareil méthodologique efficace lui permettant de s'imposer comme discipline à part entière. Consacré à la notion de formule, définie dès les premières lignes comme «un ensemble de formulations qui, du fait de leurs emplois à un moment donné et dans un espace public donné, cristallisent des enjeux politiques et sociaux que ces expressions contribuent dans le même temps à construire ", il a une visée théorique et méthodologique et s'adresse en particulier aux chercheurs et étudiants en sciences du langage et sciences de l'information et de la communication qui orientent leurs travaux vers l'analyse des discours politiques, médiatiques ou institutionnels. Ce livre figure d'ailleurs sans aucun doute parmi les « incontournables » pour tous ceux qui se lancent dans une étude du discours médiatique en prenant pour entrée le mot. La notion de formule telle qu'elle est théorisée par Alice Krieg-Planque s'impose tout à la fois comme une catégorie d'analyse opératoire pour les discours publicisés, comme une notion qui s'inscrit dans une analyse du discours critique et attentive aux matérialités linguistiques, enfin comme une notion qui procède d'une analyse discursive et langagière de l'espace public. Il faut 